\title{
EFECTO DEL FUEGO EN LA ESTRUCTURA POBLACIONAL Y LA REGENERACIÓN DE DOS ESPECIES DE ENCINOS (QueRCUS LIEBMANII OeRST. y QUeRCuS magnolilfolia Née) en la región de La Montaña (Guerrero), MéXico
}

\author{
Víctor M. Peña-Ramírez y Consuelo Bonfil ${ }^{1}$ \\ Departamento de Ecología y Recursos Naturales, Facultad de Ciencias, Universidad Nacional Autónoma de \\ México, México 04510, D.F., México.
}

1Autor para la correspondencia; Tel: 5622-4912, correo-e: cbs@fciencias.unam.mx

\begin{abstract}
Resumen: En este trabajo se analizó el efecto de los incendios superficiales en la estructura poblacional y la cobertura de dos especies arbóreas: Quercus liebmanii y Q. magnoliifolia, en San José Lagunas, Guerrero. Para cada especie se seleccionaron dos sitios en los cuales se registraron alrededor de 350 individuos por especie; éstos fueron clasificados en cuatro categorías de tamaño. Durante el periodo de estudio (1993-95) se presentaron incendios superficiales que afectaron a los cuatro sitios. Como resultado de éstos disminuyó la altura de los dos estadios juveniles, lo que produjo un aumento en la frecuencia relativa de los individuos más pequeños. La frecuencia de los árboles adultos se mantuvo sin cambios. El análisis de la cobertura mostró que las plántulas se recuperaron fácilmente después del fuego, mientras que la cobertura de los juveniles en general se redujo y la de los árboles adultos se recuperó en todos los casos. Se concluye que aunque ambas especies pueden tolerar incendios superficiales, cuando la frecuencia de éstos es muy alta, como ocurre actualmente en la zona, la regeneración de sus poblaciones puede verse inhibida.
\end{abstract}

Palabras clave: Quercus, fuego, regeneración, supervivencia, crecimiento.

\begin{abstract}
This study analyzed the effect of superficial fires on population structure and individual crown area of two tree species: Quercus liebmanii and Q. magnoliifolia, in San José Lagunas, Guerrero, southern Mexico. For each species two sites were selected, in which $c a .350$ individuals were recorded per species. Individuals were classified in four size categories. During the study period (1993-95), superficial fires occurred at all four sites. As a result, the relative frequency of the smallest plants increased due to height reductions among juveniles, while there were no changes in adult tree frequency. Analysis of changes in crown cover showed that seedlings and adult trees recovered from fire, while there were reductions in crown cover in the juvenile categories. It is concluded that although both species tolerate fires, the high frequency fire regimen prevailing in the study area may inhibit the regeneration of their populations.

Key words: Quercus, fire, regeneration, survival, growth.
\end{abstract}

A partir de que el hombre empezó a usar el fuego, la frecuencia de los incendios aumentó considerablemente, lo que ha causado modificaciones importantes en el desarrollo de los ecosistemas forestales en los últimos 10,000 años (Komarek, 1974; Fuller, 1991; Kozlowski et al., 1991; Bratton y Miller, 1994; Rodríguez, 1996). Los incendios han sido utilizados con fines de caza, aclareo de bosques para destinarlos a la agricultura, mejoramiento de pastos para el ganado, producción de ceniza para fertilizar el suelo y para favorecer a algunas especies sobre otras (Kozlowski et al., 1991; Rodríguez, 1996).
En la literatura forestal generalmente se reconocen tres tipos de incendios (Ahlgren y Ahlgren, 1960; Kozlowski et al., 1991; Retana, 1996; Rodríguez, 1996; Sosa et al., 1999). (1) Los incendios subterráneos, que se propagan bajo la superficie; éstos queman la materia orgánica del suelo y las raíces, y además son lentos y carecen de flama, por lo que son difíciles de detectar. (2) Los incendios superficiales, que consumen los restos vegetales del suelo y se propagan quemando las hierbas y arbustos del sotobosque, y pueden afectar a los troncos de los árboles adultos; éstos son los incendios más frecuentes en México (poco más del 90\%, 
Sosa et al., 1999). (3) Los incendios de copa, que se presentan en bosques densos, donde el fuego se propaga de una copa a otra; son los más rápidos y pueden consumir totalmente el arbolado, y en muchos casos se originan a partir de un incendio superficial.

La temperatura del frente de los incendios superficiales normalmente fluctúa entre 200 y $300^{\circ} \mathrm{C}$, aunque puede llegar hasta $500^{\circ} \mathrm{C}$. Sin embargo, decrece en las capas internas del suelo y raramente excede $100^{\circ} \mathrm{C}$ por debajo de una profundidad de $3 \mathrm{~cm}$ (Christensen, 1985). El fuego puede afectar las propiedades del suelo en función de la intensidad del incendio, la humedad del suelo, la cobertura vegetal, la topografía y el clima (Ahlgren y Ahlgren, 1960; Fuller, 1991; Rodríguez, 1996). Después de un incendio el suelo puede reducir su capacidad de almacenamiento de agua (debido a las capas repelentes que se desarrollan) y puede favorecerse la compactación, la erosión y la fluctuación de la temperatura superficial (Kozlowski et al., 1991). Los suelos pierden nutrimentos por volatilización, formación de ceniza, erosión y lixiviación (Viro, 1974; Kozlowski et al., 1991; Rodríguez, 1996).

Algunas especies han desarrollado adaptaciones que les permiten sobrevivir al fuego en ambientes propensos a incendiarse, aunque la temperatura es letal para los tejidos vivos cuando alcanza valores de entre 43 y $59^{\circ} \mathrm{C}$ (Hare, 1961 en Plumb, 1980). Existen básicamente dos mecanismos por los que el bosque se regenera después de un incendio: (1) la diseminación y posterior germinación de semillas, que puede verse favorecida por el fuego, como en el caso de los pinos Pinus halepensis Mill., P. pseudostrobus Lindl. y P. patula Schlecht. et Cham., y (2) la formación de rebrotes, que es común en especies de los géneros Quercus, Arbutus y Erica, entre otros (Ahlgren y Ahlgren, 1960; Ahlgren, 1974; Griffin, 1980; Negreros y Snook, 1984; Sánchez, 1986; McCreary et al., 1991; Gill, 1981 en McGee, 1995; Lloret et al., 1996; Zavala y García, 1997; Zavala et al., 2000).

El fuego ha sido el principal factor de disturbio que ha modelado y mantenido los ambientes en que prosperan los encinos (Lorimer, 1985; Abrams, 1992). Los fuegos recurrentes destruyen a las semillas enterradas en el suelo del bosque y por lo tanto eliminan o reducen la competencia post-incendio; también reducen el dosel y la densidad del sotobosque, lo que aumenta la cantidad de luz que llega al suelo y reduce la competencia (Johnson et al., 2002). En estas condiciones los encinos se ven favorecidos, ya que poseen una corteza gruesa que aísla del calor a los tejidos vivos, y muestran una gran capacidad de rebrotar cuando se produce daño o muerte de la parte aérea. Sin embargo, existen diferencias en la tolerancia al fuego entre las diversas especies del género (Plumb, 1980; Grimm, 1984), las cuales se relacionan con características como el grosor, el tipo y la composición de la corteza. Otros factores que pueden influir en la resistencia al calor son la edad, el diámetro y la altura del árbol (Plumb, 1980; Haggerty, 1991).
En los encinos, la pérdida de tallos ocasionada por fuego libera la latencia de las yemas que se encuentran bajo la corteza. Las yemas latentes, localizadas en las axilas de las ramillas, están conectadas a la parte interna del tronco, por lo que estas especies rebrotan fácilmente, ya sea a partir de las yemas de las ramas, si el incendio es ligero, o de las yemas localizadas en la unión entre la raíz y el tallo, cuando el incendio destruye la parte aérea (Kozlowski, 1971). Sin embargo, la capacidad de rebrotar en respuesta a los disturbios también difiere entre especies, y algunas se consideran más flexibles que otras en su capacidad de regenerarse ya sea por semillas o por rebrotes (Johnson $e t$ al., 2002). La regeneración por semillas no es común después de los incendios, porque las bellotas son sumamente sensibles al calor y pierden su viabilidad rápidamente (Plumb, 1980; Bonfil y Soberón, 1999).

Se han hecho estimaciones de que el $97 \%$ de los incendios forestales en México son generados por el hombre y que 54\% de éstos se deben a actividades agropecuarias (Sosa et al., 1999). Su extensión e intensidad dependen de las condiciones climáticas y meteorológicas (como temperatura y humedad relativa del aire, velocidad y turbulencia del viento), de la topografía y de la cantidad y características del combustible. De 1970 a 1999 se ha registrado un promedio anual de 6,559 incendios (poco más del $90 \%$ de ellos superficiales), con una superficie afectada de 222,656 ha (con una media de 32 ha por incendio, SEMARNAP, 1998; Sosa et al., 1999). En particular, las cifras recientes (1983-1999) colocan al estado de Guerrero como una de las entidades con mayor número de incendios y superficie afectada ( 260 incendios por año y 8,612 ha SEMARNAP, 1990, 1995, 1999).

A pesar de la importancia de los incendios en la dinámica de los ecosistemas forestales de México, han sido relativamente pocos los estudios referentes a este tema. Resulta relevante, por tanto, conocer la forma en que los incendios superficiales modelan la estructura y la regeneración de los bosques de encino y pino-encino, ya que el género Quercus presenta una alta diversidad en México (Zavala, 1990; Bonfil y Valencia, 1993; Nixon, 1993a) y sus bosques cubren grandes extensiones (Masera et al., 1997), a pesar de lo cual se conoce muy poco sobre la respuesta de las diferentes especies al fuego.

El objetivo de este estudio fue analizar el efecto del fuego en la estructura poblacional, así como en la cobertura de dos especies de encinos: Quercus liebmanii Oersted y Quercus magnoliifolia Née, en una localidad de la región de La Montaña del estado de Guerrero. Adicionalmente, y con el fin de tener un marco de referencia que permitiera evaluar la importancia relativa de estas especies en sus respectivas comunidades, así como la magnitud de los cambios originados por el fuego, se analizaron la estructura y la composición de la vegetación arbórea en los bosques de Quercus y PinusQuercus en los que dominan respectivamente dichas especies. 


\section{Materiales y métodos}

Zona de estudio. La comunidad de San José Lagunas se localiza en Alcozauca (uno los 17 municipios que constituyen la región de La Montaña), en el oriente del estado de Guerrero. Se localiza entre las latitudes $17^{\circ} 15^{\prime}$ y $17^{\circ} 30^{\prime} \mathrm{N}$ y las longitudes $98^{\circ} 18^{\prime}$ y $98^{\circ} 30^{\prime} \mathrm{O}$. Colinda al norte con el municipio de Tlalixtaquilla, al este con el estado de Oaxaca, al oeste con los municipios de Tlapa y Xalpatláhuac y al sur con el de Metlatonoc (Arias, 1993; Toledo, 1994).

El municipio cuenta con 15,058 habitantes, de los cuales $56.8 \%$ son menores de 20 años. El 93\% de la población mayor de cinco años de edad habla alguna lengua indígena y el $45 \%$ es monolingüe. Los mixtecos son el grupo más numeroso, con aproximadamente el $98 \%$ de la población indígena (INEGI, 1991). Las principales actividades económicas en la región son la agricultura, la ganadería nómada y de traspatio, la recolección de insectos (para alimento) y de plantas silvestres y la extracción de leña para consumo doméstico, así como las pequeñas industrias rurales, como el aprovechamiento de madera para muebles, casas y herramientas, la elaboración de artesanías y, en menor medida, el comercio, la educación y la administración pública (Toledo, 1994).

El municipio de Alcozauca se localiza en la cuenca del río Balsas, en la Sierra Madre del Sur. El territorio del municipio se divide en dos cuencas mayores: la del río Alcozauca, que fluye hacia el norte, con 20,978 ha (aproximadamente la mitad de la superficie total), y la cuenca del río Igualita, que abarca tres subcuencas. Ambos ríos desembocan en el río Tlapaneco, que es un afluente del Balsas. También se encuentran dos pequeños cuerpos lagunares en las comunidades de San José Lagunas y San Nicolás Zoyatlán (Toledo, 1994). El clima es (A)Cw(w)a(i')g, que corresponde a un clima semicálido, subhúmedo, con lluvias en verano (García, 1987). La temperatura media anual es de $20.4^{\circ} \mathrm{C}$ y no se presentan heladas. El mes más cálido es mayo y el más frío, enero. El promedio de precipitación total anual es de $846 \mathrm{~mm}$, con una temporada seca de noviembre a abril y una de lluvias que va de mayo a octubre. Los suelos más frecuentes son los leptosoles, que generalmente tienen una profundidad menor a los $10 \mathrm{~cm}, \mathrm{y}$ los regosoles.

Como consecuencia de la gran diversidad climática, geomorfológica y topográfica, en Alcozauca existen cuatro asociaciones vegetales (Toledo, 1994):

Bosque de Pinus-Quercus.- Incluye a dos asociaciones: la primera se encuentra sobre laderas con suelos derivados de tobas y brechas volcánicas ácidas, en elevaciones de entre 1,700 y 2,200 m. Las especies dominantes son: Pinus montezumae Lamb., Quercus magnoliifolia Née, Lysiloma acapulcense (Kunth) Benth. y Arbutus sp. La segunda asociación se desarrolla sobre laderas con suelos derivados de rocas cuarcíticas, como las areniscas, lutitas y conglomerados. Las especies dominantes son Pinus pringlei
Shaw, Quercus magnoliifolia y Quercus urbanii Trel.

Bosque de Quercus.- Se trata de un bosque dominado por Quercus liebmanii Oerst. Esta especie se desarrolla sobre laderas de roca caliza o caliza con yeso, entre 1,700 y 2,100 $\mathrm{m}$ de altitud. Las especies acompañantes incluyen a la palma Brahea dulcis (Kunth) Mart., Quercus conspersa Benth., Piscidia grandiflora (Donn.Sm.) Blake, Juniperus flaccida Schltdl., Diphysa suberosa Wats. y Lonchocarpus sp., entre otras.

Bosque tropical caducifolio.- Se desarrolla en regiones cálidas y semicálidas, entre los 1,250 y 1,350 m de altitud, sobre yesos, lutitas y brechas volcánicas. Las especies dominantes son Lysiloma acapulcense, Lysiloma divaricatum (Jacq.) J.F.Macbr., Bursera lancifolia Engl., Bursera morelense Ramírez, Bursera longipes Rose, Bursera submoniliformis Engl., Bursera vejar-vazquezii Miranda, Heliocarpus tomentosus Turcz., Vitex mollis Kunth y Plumeria acutifolia Poir.

Vegetación ribereña.- Se limita a la ribera del río Salado. Las especies dominantes son Astianthus viminalis (Kunth) Baill., Salix humboldtiana Willd., Ficus cotinifolia Kunth, Ficus petiolaris Kunth, Ficus segoviae Miq. y en menor medida, Taxodium mucronatum Ten.

Especies de estudio. Las dos especies en las que se enfoca el presente estudio son Quercus liebmanii y Quercus magnoliifolia. Ambas pertenecen al subgénero Quercus sensu Nixon (1993b). A continuación se ofrece una breve descripción de cada una de ellas.

Quercus liebmanii es un árbol que alcanza hasta $10 \mathrm{~m}$ de alto y $0.7 \mathrm{~m}$ de diámetro. Pierde las hojas y florece de marzo a mayo, y sus frutos maduran de junio a agosto. Forma bosques casi puros, o bien se asocia con Quercus castanea Née, Quercus glaucoides Mart. et Gal., Quercus splendens Née y en la zona de estudio con Quercus conspersa. Además del estado del Guerrero, su distribución comprende Colima, Jalisco, México, Michoacán, Nayarit, Oaxaca, Puebla y Sinaloa. Su madera se utiliza principalmente para carbón y leña (González-Villareal, 1986; Valencia, 1995).

Quercus magnoliifolia también es un árbol de 5 a $10 \mathrm{~m}$ de alto, aunque en las montañas próximas a la costa su altura llega hasta $25 \mathrm{~m}$ y el diámetro de su tronco hasta $0.5 \mathrm{~m}$. Durante marzo y abril pierde el follaje y florece; los frutos maduran de mayo a julio. En bosques de Quercus puede encontrarse asociado con Quercus acutifolia Née, Quercus castanea y Quercus glaucoides, y en bosques de PinusQuercus con Juniperus flaccida, Pinus michoacana Mart., Pinus lawsoni Roezl y Arbustus xalapensis Sarg. Además de Guerrero, se localiza en Sinaloa, Nayarit, Jalisco, Colima, Michoacán, Oaxaca y América Central. Su madera se utiliza como carbón, leña, postería y horcones; su follaje para techos rústicos de viviendas del campo (González-Villareal, 1986; Valencia, 1995). 
Víctor M. Peña-Ramírez y Consuelo Bonfil

Cuadro 1. Características de los cuatro sitios de estudio.

\begin{tabular}{|c|c|c|c|c|}
\hline & \multicolumn{2}{|c|}{$\begin{array}{l}\text { Bosque de Quercus } \\
\text { (Quercus liebmanii) }\end{array}$} & \multicolumn{2}{|c|}{$\begin{array}{l}\text { Bosque de Pinus-Quercus } \\
\text { (Quercus magnoliifolia) }\end{array}$} \\
\hline & Sitio 1 & Sitio 2 & Sitio 3 & Sitio 4 \\
\hline Orientación & SW & NE & E-NE & $\mathrm{N}-\mathrm{NE}$ \\
\hline Pendiente promedio & $30^{\circ}$ & $26^{0}$ & $35^{0}$ & $32^{0}$ \\
\hline Altitud (m snm) & 1,800 & 1,800 & 2,000 & 2,000 \\
\hline Material parental & Calizas y yesos & Calizas y yesos & $\begin{array}{l}\text { Areniscas y } \\
\text { conglomerados } \\
\text { cuarcíticos }\end{array}$ & $\begin{array}{c}\text { Areniscas y } \\
\text { conglomerados } \\
\text { cuarcíticos }\end{array}$ \\
\hline
\end{tabular}

Estructura de la comunidad. Con base en la información obtenida durante recorridos previos por la zona, se seleccionaron dos sitios en el bosque de Quercus y otros dos en el bosque de Pinus-Quercus. En cada caso los dos sitios seleccionados fueron similares entre sí, aunque con diferente orientación. Quercus liebmanii únicamente se distribuye en la primera asociación vegetal (sitios 1 y 2) y Quercus magnoliifolia en la segunda (sitios 3 y 4). En cada sitio se registraron los siguientes datos: orientación de la ladera, material parental, altitud y pendiente. La información resumida se presenta en el cuadro 1.

En cada sitio se colocaron cinco cuadros de $10 \times 10 \mathrm{~m}$ (500 $\mathrm{m}^{2}$ en total por sitio); este tamaño de cuadro (o subcuadro, en su caso) ha sido usado en otros estudios de vegetación forestal en México (Sarukhán, 1968; Meave del Castillo, 1990). Los cuadros se ubicaron eligiendo puntos al azar a lo largo de un transecto de $150 \mathrm{~m}$ paralelo a la pendiente.

En cada cuadro se registraron los árboles de todas las especies presentes, definidos como aquellos individuos con alturas mayores a $3 \mathrm{~m}$, todos los cuales tienen como mínimo un perímetro a la altura del pecho (PAP) de $10 \mathrm{~cm}(3.18 \mathrm{~cm}$ DAP), anotando su altura, cobertura y perímetro a la altura del pecho. Esta última variable fue empleada para calcular el área basal, ya que los árboles no presentaban contrafuertes o engrosamiento en la base. En el caso de la palma Brahea dulcis se registró la altura de los individuos con alturas $>2$ $\mathrm{m}$, dado que ésta es una especie importante cuya forma de crecimiento no permite aplicar los criterios antes establecidos.

El índice estimado en cada sitio para comparar la importancia relativa de Quercus liebmanii y $Q$. magnoliifolia con respecto a las demás especies arbóreas, fue el índice de valor de importancia relativa (IVI) propuesto por Curtis y McIntosh (1951):

$$
I V I=(\text { Drel })+(\text { Frel })+(\text { Drel })
$$

donde Drel, Frel y Domrel son los valores relativos de la densidad, la frecuencia y la dominancia, respectivamente.

Efecto del fuego en la estructura poblacional de los encinos. Para conocer la estructura poblacional y analizar el efecto del fuego sobre ella para ambas especies de encinos se definieron las siguientes categorías de tamaño: (1) plántulas, (2) juveniles 1, (3) juveniles 2, y (4) árboles. Como plántulas fueron considerados los individuos con una altura $\leq 50 \mathrm{~cm}$, incluyendo tanto a las plantas provenientes de semillas que germinaron recientemente (plántulas sensu stricto) como a rebrotes de mayor edad que no han rebasado esta altura. En la categoría juveniles 1 se ubicaron los individuos con una altura $>50 \mathrm{~cm} \mathrm{y} \leq 1 \mathrm{~m}$, mientras que en juveniles 2 fueron incluidos los individuos con una altura $>1 \mathrm{~m} \mathrm{y} \leq 3 \mathrm{~m}$. Finalmente, los árboles quedaron definidos como los individuos con una altura $>3 \mathrm{~m}$ y un perímetro a la altura del pecho $(\mathrm{PAP}) \geq 10 \mathrm{~cm}(\mathrm{DAP} \geq 3.2 \mathrm{~cm})$.

En cada cuadro de $10 \times 10$ se registraron todos los individuos presentes. Para los árboles se usaron los datos obtenidos por el procedimiento mencionado en el apartado anterior. Aunque es posible que en esta categoría, formada con base en el tamaño, se incluyan tanto a individuos reproductivos como a individuos pre-reproductivos, observaciones aisladas sugieren que los individuos reproductivos de menor tamaño tienen estas dimensiones, y dado que no todos los años hay producción de frutos, se decidió usar este criterio práctico. Para todos los árboles con alturas $>3 \mathrm{~m}$ se registraron valores de PAP $>10 \mathrm{~cm}$, por lo que todos los individuos de la población quedan comprendidos en alguna de estas categorías. Para los juveniles se registró el número de individuos, la altura y la cobertura de cada uno en los mismos cuadros. En el caso de las plántulas, se colocaron sistemáticamente cinco cuadros de $1 \mathrm{~m}^{2}$ en cada esquina y en el centro del cuadro mayor, y se 
Cuadro 2. Fechas en que se realizaron las mediciones y en que se presentaron los incendios (A: árboles, J: juveniles, P: plántulas)

\begin{tabular}{|c|c|c|c|c|}
\hline & \multicolumn{2}{|c|}{$\begin{array}{l}\text { Bosque de Quercus } \\
\text { (Quercus liebmanii) }\end{array}$} & \multicolumn{2}{|c|}{$\begin{array}{l}\text { Bosque de Pinus-Quercus } \\
\text { (Quercus magnoliifolia) }\end{array}$} \\
\hline & Sitio 1 & Sitio 2 & Sitio 3 & Sitio 4 \\
\hline Primera medición & jun. 1993 (A) & jun. 1993 (A) & nov. $1993(\mathrm{~A}, \mathrm{P})$ & nov. de 1993 (A) \\
\hline Segunda medición & feb. $1994(J, P)$ & feb. $1994(J, P)$ & feb. 1994 (J) & $\begin{array}{l}\text { feb. } 1994(\mathrm{P}) \\
\text { may. } 1994(\mathrm{~J})\end{array}$ \\
\hline Tercera medición & nov.1994 (J, P) & nov. 1994 (J, P) & nov. $1994(\mathrm{~J}, \mathrm{P})$ & nov. $1994(J, P)$ \\
\hline Cuarta medición & jul. $1995(\mathrm{~A}, \mathrm{~J}, \mathrm{P})$ & jul. $1995(\mathrm{~A}, \mathrm{~J}, \mathrm{P})$ & jul. $1995(\mathrm{~A}, \mathrm{~J}, \mathrm{P})$ & jul. $1995(A, J, P)$ \\
\hline Fecha del incendio & mayo de 1994 & mayo de 1995 & mayo de 1995 & mayo de 1995 \\
\hline
\end{tabular}

registraron la altura, el diámetro basal y la cobertura de cada plántula presente. Cada individuo fue etiquetado y su ubicación en el cuadro registrada, para facilitar su posterior localización.

Las fechas en que se realizaron las sucesivas mediciones se muestran en el cuadro 2. Aproximadamente un año después del primer muestreo se realizó una segunda medición con el objetivo de registrar la supervivencia y el crecimiento de los individuos. Finalmente, una tercera medición se realizó a los dos años del registro inicial. Sin embargo, en cada uno de los sitios muestreados se presentó accidentalmente un incendio superficial, cuyas fechas de ocurrencia se presentan en el mismo cuadro.
Empleando los datos de frecuencia de las cuatro categorías de tamaño en cada sitio, se realizó una prueba de $\mathrm{G}$ (de bondad de ajuste; Sokal y Rohlf, 1995) para comparar las distribuciones de frecuencias antes y después de haberse presentado el incendio, con el propósito de detectar posibles cambios en la estructura poblacional de ambas especies.

Finalmente, en cada especie se analizó la cobertura (área de la copa por individuo) de cada categoría de tamaño por separado (eliminando los datos con cobertura cero), por medio de un análisis de varianza de dos vías (Sokal y Rohlf, 1995), en el que los factores fueron: "sitio" (con dos niveles) y "tiempo" (que incluye dos o tres fechas, dos de las cuales corresponden a las medidas registradas antes y después de

Cuadro 3. Valores de importancia relativa del estrato arbóreo del bosque de Quercus liebmanii.

\begin{tabular}{lcccccc}
\hline Especie & Sitio & $\begin{array}{c}\text { Número de } \\
\text { individuos }\end{array}$ & $\begin{array}{c}\text { Densidad } \\
\text { relativa } \\
(\mathrm{a})\end{array}$ & $\begin{array}{c}\text { Frecuencia } \\
\text { relativa } \\
(\mathrm{b})\end{array}$ & $\begin{array}{c}\text { Dominancia } \\
\text { relativa } \\
(\mathrm{c})\end{array}$ & $\begin{array}{c}\text { Valor de } \\
\text { importancia relativa } \\
(\mathrm{a}+\mathrm{b}+\mathrm{c})\end{array}$ \\
\hline $\begin{array}{l}\text { Quercus } \\
\text { liebmanii }\end{array}$ & 1 & 37 & 84.09 & 50 & 80.90 & 214.99 \\
Brahea dulcis & 2 & 45 & 69.23 & 50 & 61.45 & 180.68 \\
& 1 & 5 & 11.36 & 30 & 9.53 & 50.89 \\
Lysiloma & 2 & 20 & 30.77 & 50 & 38.54 & 119.31 \\
acapulcense & 1 & 1 & 2.27 & 10 & 2.52 & 14.79 \\
Lysiloma sp. & 2 & 0 & 0 & 0 & 0 & 0 \\
& 1 & 1 & 2.27 & 10 & 7.04 & 19.31 \\
& 2 & 0 & 0 & 0 & 0 & 0 \\
\hline
\end{tabular}


Víctor M. PeÑA-Ramírez y Consuelo BonfIL

Cuadro 4. Valores de importancia relativa del estrato arbóreo del bosque de Pinus - Quercus magnoliifolia.

\begin{tabular}{lcccccc}
\hline \multicolumn{1}{c}{ Especie } & Sitio & $\begin{array}{c}\text { Número de } \\
\text { individuos }\end{array}$ & $\begin{array}{c}\text { Densidad } \\
\text { relativa } \\
(\mathrm{a})\end{array}$ & $\begin{array}{c}\text { Frecuencia } \\
\text { relativa } \\
(\mathrm{b})\end{array}$ & $\begin{array}{c}\text { Dominancia } \\
\text { relativa } \\
(\mathrm{c})\end{array}$ & $\begin{array}{c}\text { Valor de } \\
\text { importancia relativa } \\
(\mathrm{a}+\mathrm{b}+\mathrm{c})\end{array}$ \\
\hline Quercus magnoliifolia & 3 & 12 & 57.14 & 40 & 32.94 & 130.08 \\
& 4 & 20 & 71.43 & 100 & 51.82 & 223.25 \\
Pinus pringlei & 3 & 7 & 33.33 & 40 & 63.15 & 136.48 \\
& 4 & 3 & 10.71 & 40 & 38.88 & 89.59 \\
Pinus montezumae & 3 & 2 & 9.52 & 10 & 3.35 & 22.87 \\
& 4 & 0 & 0 & 0 & 0 & 0 \\
Lysiloma acapulcense & 3 & 0 & 0 & 0 & 0 & 0 \\
Arbutus xalapensis & 4 & 4 & 14.29 & 40 & 8.61 & 62.90 \\
& 3 & 0 & 0 & 0 & 0 & 0 \\
& 4 & 1 & 3.57 & 10 & 0.69 & 14.26 \\
\hline
\end{tabular}

que se presentara el incendio). La cobertura se calculó con la fórmula del área de una elipse:

$$
A=\left(D_{1} / 2\right)\left(D_{2} / 2\right)(\pi)
$$

donde $\mathrm{D}_{1}$ es el diámetro mayor y $\mathrm{D}_{2}$ el perpendicular a éste. El programa de cómputo empleado fue el SYSTAT 7.0.

\section{Resultados}

Estructura y composición de la vegetación arbórea. En el bosque de Quercus la especie con el valor de importancia más alto fue Quercus liebmanii. Ésta presentó un valor de importancia mayor en el sitio 1, a pesar de contar con menor número de individuos que en el sitio 2 (cuadro 3), debido a que en el primero sus troncos fueron de mayor talla y, en menor medida, a que su densidad fue alta respecto a las demás especies presentes en el sitio. La segunda especie en importancia en ambos sitios resultó ser Brahea dulcis. Esta palma exhibió un valor de importancia más alto en el sitio 2 debido a que tuvo cuatro veces más individuos que el sitio 1 . Las dos especies de leguminosas restantes tuvieron un valor de importancia por lo menos 10 veces inferior al valor de $Q$. liebmanii.

Los resultados del mismo análisis para el bosque de PinusQuercus mostraron que Pinus pringlei fue la especie con el valor de importancia más alto en el sitio 3 , a pesar de contar con menos individuos que $Q$. magnoliifolia (cuadro 4). Esto se debió a su gran área basal, pues los troncos de dichos árboles alcanzan un tamaño considerable. En el sitio 4 las dos especies de pinos ( $P$. pringlei y $P$. montezumae) tuvieron una menor representación, siendo $Q$. magnoliifolia la especie dominante. Al comparar los valores de importancia de $Q$. magnoliifolia entre sitios, se observó que en el sitio 4 fue mayor debido a una mayor densidad.
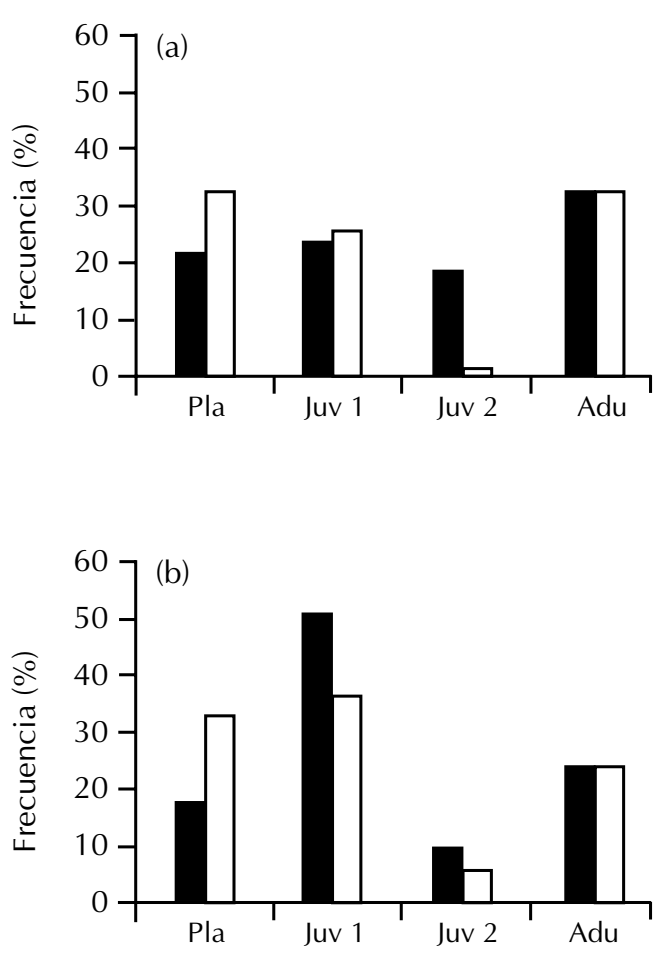

Figura 1. Distribuciones de frecuencias preincendio (barra negra) y postincendio (barra blanca) de las categorías de tamaño de Quercus liebmanii. (a) Sitio 1, (b) Sitio 2. Pla = Plántulas, Juv $1=$ Juveniles 1, Juv 2 = Juveniles 2, Adu =Adultos 


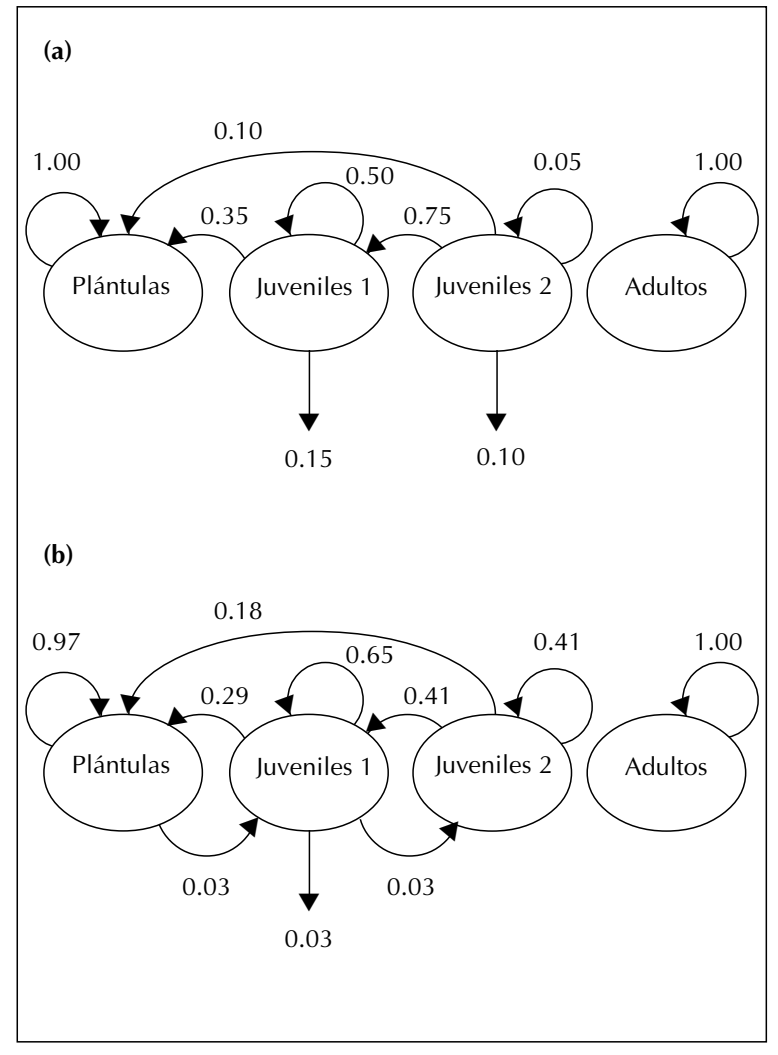

Figura 2. Diagrama de ciclo de vida de Quercus liebmanii. (a) Sitio 1, (b) Sitio 2

Resalta que las dos especies de encinos consideradas se distribuyen en ambientes diferentes y no se presentan mezcladas, a pesar de que los sitios 1 y 2 se encontraban en una elevación adyacente al cerro en que se ubicaron los sitios 3 y 4 . En los primeros domina $Q$. liebmanii. En el sitio 4 la especie dominante fue $Q$. magnoliifolia, que en el sitio 3 se encontró como especie codominante con P. pringlei. Este sitio también se distingue porque sólo en él se presentó la especie $P$. montezumae y, al contrario del sitio 4, en él no se registró la presencia de Arbutus xalapensis ni de Lysiloma acapulcense.

Efecto del fuego en la estructura poblacional. Las pruebas de $\mathrm{G}$ realizadas con el fin de comparar la distribución de frecuencias poblacionales de cada sitio antes y después de que se presentaran los incendios, mostraron diferencias altamente significativas para la población de $Q$. liebmanii en el sitio 1 ( $G=22.694, P<0.001)$, lo que confirma que el incendio (mayo de 1994) modificó la estructura de esta población (figura 1a). Las transiciones asociadas a estos cambios se muestran en la figura 2a. Destaca el hecho de que el estadio más afectado fue el de juveniles 2: después del incendio únicamente $5 \%$ de los individuos se mantuvo en esta categoría, mientras que $75 \%$ de ellos pasó a formar parte del estadio anterior, $10 \%$ disminuyó en altura lo suficiente para ser incluido en la categoría de plántulas y otro $10 \%$ sucumbió a consecuencia del incendio. En lo que respecta a la categoría de juveniles $1,50 \%$ de los individuos se mantuvo en su mismo estadio, $35 \%$ pasó al estadio de plántulas y $15 \%$ pereció. Los árboles y las plántulas se mantuvieron sin cambios en sus frecuencias y no se registró ninguna transición positiva en el periodo.

En el sitio 2 la distribución de frecuencias de la población también cambió significativamente a consecuencia del incendio $(G=14.663, P<0.01)$. Los individuos más afectados por el fuego (mayo de 1995) fueron los de ambos estadios juveniles (figura 1b). En el caso de los juveniles 2, un porcentaje igual $(41.2 \%)$ al que permaneció en la misma categoría decreció a juveniles 1 , y el resto de los individuos decreció lo suficiente como para transitar a la categoría de plántulas (figura 2b). En cuanto a los juveniles 1, la mayoría permaneció en el mismo estadio, $29 \%$ decreció, un pequeño
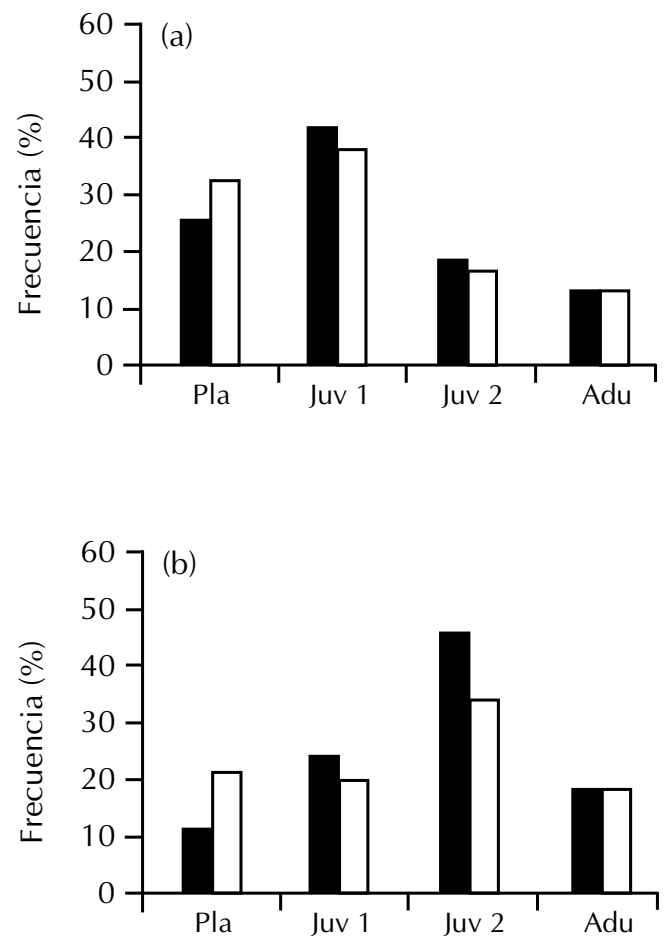

Figura 3. Distribuciones de frecuencias preincendio (barra negra) y postincendio (barra blanca) de las categorías de tamaño de Quercus magnoliifolia. (a) Sitio 3, (b) Sitio 4. Pla = Plántulas, Juv 1= Juveniles 1, Juv 2b = Juveniles 2, Adu = Adultos 


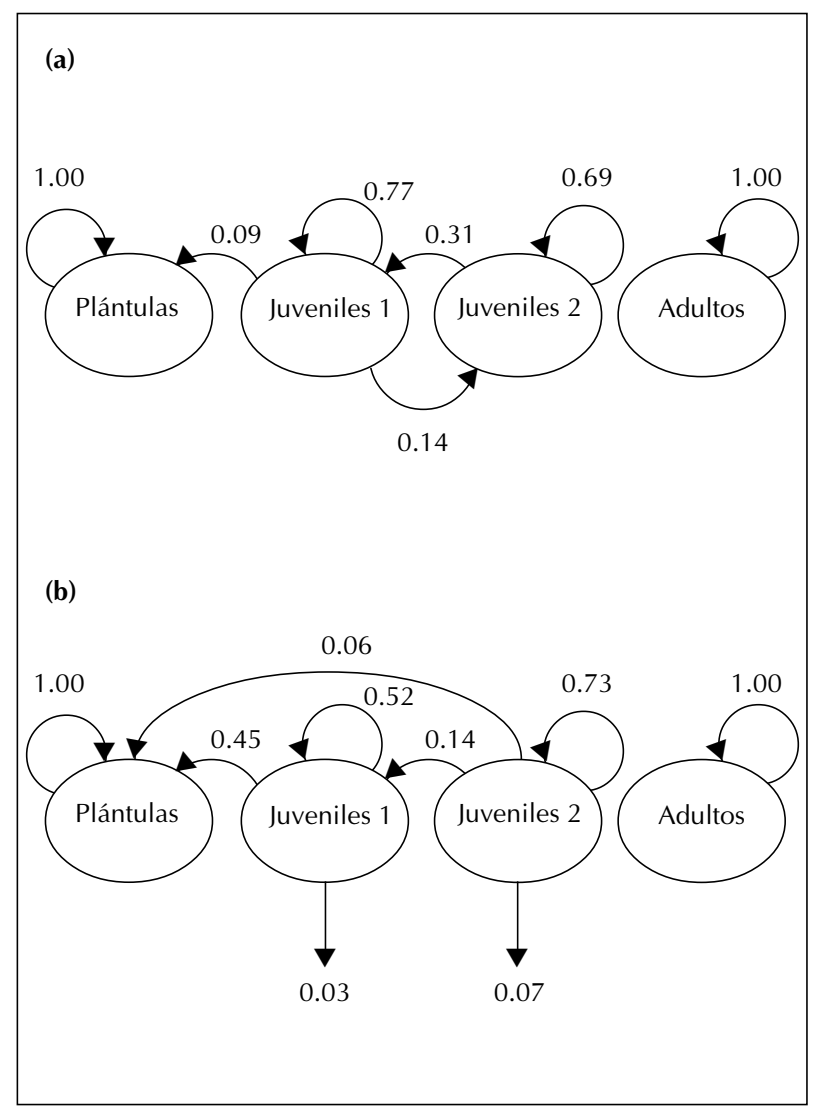

Figura 4. Diagrama de ciclo de vida de Quercus magnoliifolia. (a) Sitio 3, (b) Sitio 4. porcentaje $(3.1 \%)$ pereció y otro porcentaje igual transitó hacia el estadio juveniles 2 . Los árboles permanecieron sin cambios en sus frecuencias, al igual que la mayor parte de las plántulas, aunque de algunas de éstas crecieron y pasaron a la categoría de juveniles 1. Como en el caso anterior, el aumento en la proporción de plántulas que se observó después del fuego fue consecuencia de la disminución en el tamaño de individuos pertenecientes a los estadios juveniles (figura 1b).

En el sitio 3 no se registraron diferencias significativas $(G=0.765, P=0.90)$ entre la distribución de frecuencias de Q. magnoliifolia antes y después del incendio (mayo de 1995), y la estructura de la población se mantuvo prácticamente sin cambios (figura 3a). Destaca el hecho de que no se registró mortalidad a causa del incendio. Sin embargo, sí se dieron algunas transiciones importantes (figura 4a): por ejemplo, una tercera parte de los juveniles 2 transitó al estadio de juveniles 1. Asimismo, alrededor de $9 \%$ de los Juveniles 1 decreció. En esta categoría se registró la única transición positiva, ya que $14 \%$ de los individuos creció y transitó a la categoría de juveniles 2 . Los árboles y las plántulas permanecieron sin cambios en sus frecuencias.

En el sitio 4 la distribución de frecuencias de $Q$. magnoliifolia tampoco cambió significativamente a consecuencia del fuego $(G=6.989, P=0.10$, figura $3 b)$, aunque fue evidente que nuevamente los estadios juveniles 1 y juveniles 2 fueron afectados por el incendio (figura $4 \mathrm{~b}$ ). Aunque la mayor parte de los juveniles 2 permaneció en su misma categoría, poco más del $20 \%$ decreció, incluso hasta la categoría de plántulas, y se presentó mortalidad a consecuencia del incendio. En el caso de los juveniles 1, proporciones similares se mantuvieron en su mismo estadio

Cuadro 5. Resultados de los análisis de varianza de dos vías (S-sitio y T-tiempo) en la cobertura.

\begin{tabular}{|c|c|c|c|c|c|c|c|}
\hline & \multirow[b]{2}{*}{ Factor } & \multicolumn{3}{|c|}{ Quercus liebmanii } & \multicolumn{3}{|c|}{ Quercus mangnoliifolia } \\
\hline & & g. I. & $\mathrm{F}$ & $\mathrm{p}$ & g. I. & $\mathrm{F}$ & $\mathrm{p}$ \\
\hline \multirow[t]{3}{*}{ Plántulas } & $S$ & 1 & 0.65 & 0.42 & 1 & 3.91 & 0.05 \\
\hline & $\mathrm{T}$ & 2 & 0.05 & 0.95 & 2 & 1.68 & 0.19 \\
\hline & SxT & 2 & 0.03 & 0.97 & 2 & 0.22 & 0.80 \\
\hline \multirow[t]{3}{*}{ Juveniles 1} & $\mathrm{~s}$ & 1 & 2.09 & 0.15 & 1 & 0.21 & 0.65 \\
\hline & $\mathrm{T}$ & 2 & 9.35 & $<0.001$ & 1 & 1.70 & 0.004 \\
\hline & SxT & 2 & 14.53 & $<0.001$ & 1 & 2.01 & 0.16 \\
\hline \multirow[t]{3}{*}{ Juveniles 2} & $S$ & 1 & 0.52 & 0.82 & 1 & 3.08 & 0.08 \\
\hline & $\mathrm{T}$ & 2 & 2.80 & 0.06 & 1 & 3.13 & 0.08 \\
\hline & SxT & 2 & 6.61 & 0.002 & 1 & 0.56 & 0.45 \\
\hline \multirow[t]{3}{*}{ Árboles } & $S$ & 1 & 37.18 & $<0.001$ & 1 & 11.47 & 0.001 \\
\hline & $\mathrm{T}$ & 1 & 0.02 & 0.88 & 1 & 2.15 & 0.15 \\
\hline & SxT & 1 & 1.63 & 0.20 & 1 & 1.24 & 0.27 \\
\hline
\end{tabular}


Efecto del fuego en dos especies de encinos en La Montaña, Guerrero

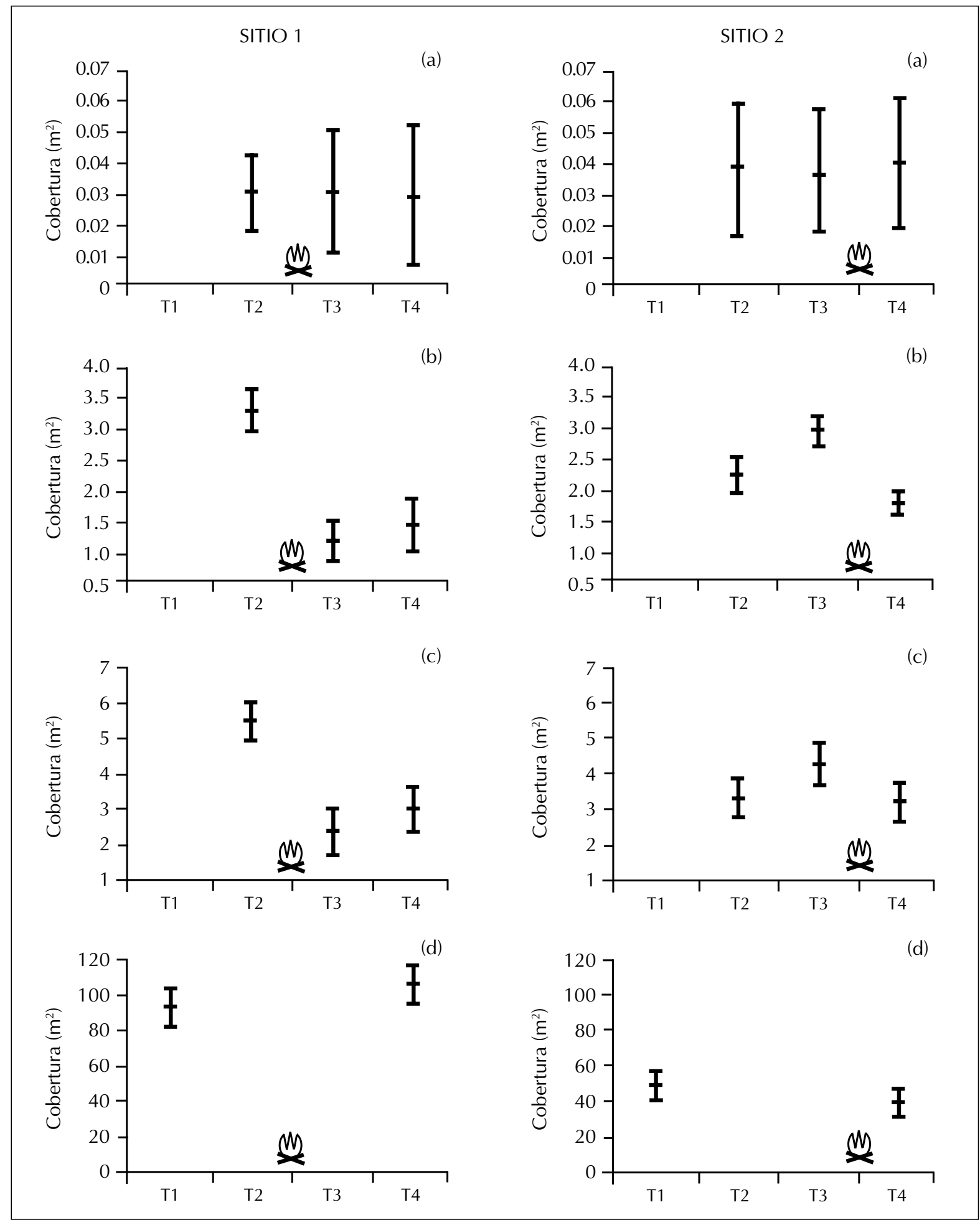

Figura 5. Cambio de la cobertura en Quercus liebmanii. (a) Plántulas, (b) Juveniles 1, (c) Juveniles 2, (d) Adultos. El símbolo de fuego señala la fecha en que se presentó el incendio. 
Víctor M. Peña-RamíreZ y Consuelo Bonfil

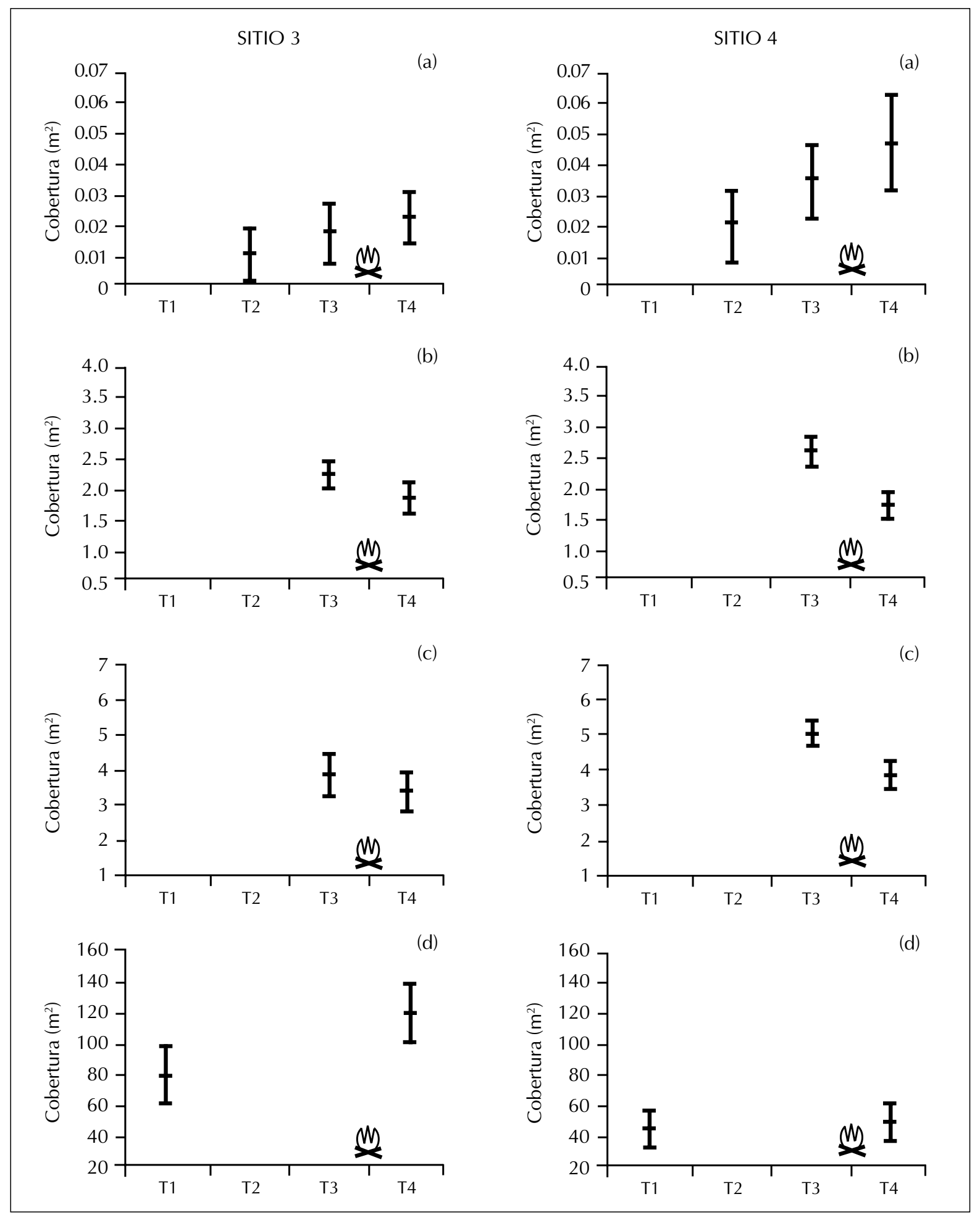

Figura 6. Cambio de la cobertura en Quercus magnoliifolia. (a) Plántulas, (b) Juveniles 1, (c) Juveniles 2, (d) Adultos. El símbolo de fuego señala la fecha en que se presentó el incendio. 
y decrecieron, y 3\% pereció. Las frecuencias de los árboles y las plántulas permanecieron sin cambios, y no se registraron transiciones positivas (figura $4 b$ ).

Efecto del fuego en la cobertura. Los análisis de varianza de dos vías permitieron analizar el efecto de los factores sitio (1 y 2 para Quercus liebmanii; 3 y 4 para $Q$. magnoliifolia), y tiempo (que puede equipararse a "incendio", ya que incluye los registros correspondientes a los tiempos anterior y posterior al fuego), y la interacción entre ambos factores, en la cobertura de cada categoría de tamaño (cuadro 5).

En el caso de las plántulas de Q. liebmanii no se registró un efecto significativo de los factores analizados y la cobertura promedio después de año y medio fue prácticamente la misma que al principio del estudio (cuadro 5, figura 5a). En ambos sitios, poco después (seis y dos meses, respectivamente) de que el fuego quemara completamente los tallos, las plántulas recuperaron su cobertura. El fuego tampoco afectó a la cobertura de las plántulas de Q. magnoliifolia (cuadro 5), aunque el efecto del sitio resultó significativo, debido a que los individuos del sitio 4 tuvieron una cobertura promedio mayor que los del sitio 3, tanto antes como después del incendio (figura 6a).

Para el estadio juveniles 1 de $Q$. liebmanii, el tiempo y la interacción sitio-tiempo resultaron significativas (cuadro 5). Se presentó una disminución en las coberturas promedio como resultado del fuego. En el sitio 1, el incendio produjo que la cobertura de los individuos disminuyera aproximadamente $64 \%$, mientras que en el sitio 2 éstos recuperaron en promedio más de la mitad de su cobertura original dos meses después del incendio (figura $5 b$ ).

El incendio afectó también la cobertura de los juveniles 1 de $Q$. magnoliifolia (efecto significativo del tiempo en el cuadro 5). En ambos sitios se presentó una disminución de la cobertura a consecuencia del incendio, aunque también se observó una buena recuperación $(\approx 70 \%)$ tan sólo dos meses después (figura 6b). En ambos estadios juveniles de esta especie únicamente se registró la cobertura en dos fechas (antes y después del incendio), dado que en la época seca de 1994 (febrero-mayo) la medición coincidió con la fase fenológica en que los individuos pierden las hojas como una respuesta adaptativa a la sequía.

En los juveniles 2 de $Q$. liebmanii sólo la interacción sitio $x$ tiempo tuvo un efecto significativo sobre la cobertura, aunque la probabilidad asociada al tiempo (incendio) se encuentra cerca del nivel de significancia (cuadro 5). Los valores promedio de la cobertura mostraron la misma tendencia que en el caso anterior: una reducción en la cobertura de cada sitio en la fecha inmediatamente después del incendio y una recuperación posterior (que se puede observar al menos en el sitio 1, figura 5c), que sin embargo no alcanzó a reponer la cobertura original pre-incendio. Para los juveniles 2 de $Q$. magnoliifolia, a diferencia de $Q$. liebmanii, ningún factor resultó significativo (cuadro 5), aunque en ambos sitos se redujo la cobertura (figura 6c).

Finalmente, en los árboles de ambas especies sólo el factor sitio tuvo un efecto significativo sobre la cobertura. Para $Q$. liebmanii esto se debe a que el sitio 1 tuvo en promedio árboles con coberturas mayores de manera consistente, mientras que para $Q$. magnoliifolia esto sucedió en el sitio 3 (figuras $5 \mathrm{~d}$ y $6 \mathrm{~d}$ ).

\section{Discusión}

Estructura y composición de la vegetación arbórea. Diversas investigaciones han mostrado que la presencia de bosques de Quercus se encuentra asociada a los disturbios, en particular a los incendios recurrentes (Johnson et al., 2002). En varias regiones de los Estados Unidos de Norteamérica el control de incendios ha causado el reemplazo de los encinos por especies más tolerantes a la sombra (Lorimer, 1985; Abrams y Downs 1990; Sieg y Wright, 1996), pero este fenómeno se ha registrado sobre todo en sitios mésicos (Abrams, 1992), mientras que la dinámica de los encinares secos, ya sea en ausencia de disturbios o en respuesta al incremento en la frecuencia de éstos, se conoce poco hasta la fecha.

Es probable que en la región de La Montaña la existencia de los bosques de encino o de pino encino también se relacione con una historia de fuegos periódicos, pues éstos pueden verse favorecidos por las condiciones climáticas imperantes, de altas temperaturas durante la temporada más seca del año. Sin embargo, actualmente en San José Lagunas la frecuencia de incendios es muy alta, pues el fuego (empleado en la preparación de tierras de cultivo y para la promoción de los brotes de pastos para el ganado) se sale de control con facilidad, lo que ha ocasionado que en periodos de cinco años se presenten comúnmente entre dos y tres incendios en el mismo sitio (observación personal). Es de esperarse, por tanto, que la estructura y composición, así como la dinámica que presentan los bosques de Quercus y de PinusQuercus que se localizan en los alrededores de este poblado, esté fuertemente influida por éste y otros disturbios periódicos, como la extracción de leña y el pastoreo de ganado.

En los dos sitios de bosque de Quercus (1 y 2), Q. liebmanii fue la especie dominante, seguida por la palma Brahea dulcis. Esto significa que la dinámica de la comunidad está dada en buena medida por el comportamiento demográfico de la primera especie. Aunque el número de individuos fue menor en el sitio 1 que en el sitio 2, el valor de importancia más alto de $Q$. liebmanii en el primero se debió a que los árboles tuvieron un área basal más grande (cuadro 3), lo que permite suponer que se trata de individuos con mayor edad que los del sitio 2. La disparidad en el número de individuos podría estar relacionada con la presencia de condiciones relativamente más secas en el sitio 1 (dada su exposición $\mathrm{SO})$, o con una mayor frecuencia de disturbios 
antropogénicos, como colecta de leña y pastoreo (obs. pers.). La alta importancia relativa de Brahea dulcis en ambos sitios, palma cuyas hojas coriáceas protegen a los meristemos apicales del fuego, es un indicio de que la zona es frecuentemente afectada por incendios (Toledo, 1994; Valiente-Banuet et al., 1995).

En el bosque de Pinus-Quercus se encontró una codominancia entre $P$. pringlei (dominante en el sitio 3) y $Q$. magnoliifolia (dominante en el sitio 4). Sin embargo, el sitio 3, con P. pringlei como especie dominante, refleja mejor la composición de esta comunidad vegetal (cuadro 4). Muchos pinos están asociados a estados sucesionales tempranos debido a que su establecimiento se ve favorecido en lo sitios abiertos que resultan de grandes disturbios (Rzedowski, 1978; Obieta y Sarukhán, 1981; Negreros y Snook, 1984; Sánchez, 1986; Williams y Johnson, 1990; Perry 1991; Williams y Johnson, 1992). Por el contrario, las plántulas de muchas especies de encinos son tolerantes a la sombra, y por lo general son árboles de lento crecimiento que aparecen en los estadios sucesionales más avanzados (Rzedowski, 1978; Crow, 1988; González-Espinosa et al., 1991; QuintanaAscencio et al., 1992; Zavala et al., 2000). Por ello, es muy posible que la dominancia de $P$. pringle $i$ esté relacionada con una historia de incendios presente desde épocas remotas.

Los individuos adultos de $P$. pringlei, como los de $P$. patula, $P$. montezumae, $P$. pseudostrobus y $P$. hartwegii, entre otros, poseen una corteza gruesa que evita el calentamiento interno, lo que les permite persistir después de los incendios superficiales. Además, al igual que otras especies de pinos (como $P$. contorta, $P$. radiata, $P$. patula y $P$. oocarpa) presentan conos serotinos que requieren de altas temperaturas para abrirse, por lo que la dispersión de semillas se ve favorecida por el fuego (Rebertus et al., 1989; Williams y Johnson, 1990; Perry, 1991; Williams y Johnson, 1992; Rodríguez, 1996; Keeley y Zedler, 1998). El dosel abierto, que puede resultar de un incendio superficial o de copa, brinda las condiciones de luz necesarias para el crecimiento de los pinos, cuya tasa de crecimiento en sitios abiertos es mayor que la de los encinos (Williamson y Black, 1981; Bucley, 1984; Sánchez, 1986).

Efecto del fuego en la estructura poblacional y la cobertura. En los dos sitios en donde se estudió la estructura de tamaños de la población de $Q$. liebmanii los resultados mostraron diferencias en la distribución de las frecuencias ocasionadas por el incendio. Por el contrario, la estructura de las poblaciones de $Q$. magnoliffolia se mantuvo sin cambios significativos, aunque en los dos sitios considerados se presentaron transiciones importantes, que se analizan más adelante.

En los diagramas de ciclo de vida de $Q$. liebmanii (figura 2) destaca el hecho de que en el periodo analizado dominaron las transiciones correspondientes a procesos de estasis y retrogresión (sensu Silvertown et al., 1993). La estasis fue especialmente relevante en las plántulas y los árboles, mientras que en los dos estadios juveniles la retrogresión (o decremento en talla) fue la consecuencia más relevante del fuego, con valores particularmente altos para los Juveniles 2 en ambos sitios ( $85 \%$ de los individuos en el sitio 1 y $59 \%$ en el sitio 2 ), lo que parece indicar que éste es un estadio particularmente susceptible al fuego. Los Juveniles 1 tuvieron un comportamiento muy similar, con magnitudes de retrogresión menores, pero todavía muy importantes (figuras $2 \mathrm{a}$ y $2 \mathrm{~b}$ ) . Destaca también la baja frecuencia con que se presentaron transiciones de crecimiento. De seis transiciones posibles de crecimiento (considerando ambos sitios), sólo se presentaron dos. El valor de ambas fue relativamente bajo; en el caso de Juveniles 1 el valor es menor al registrado para la misma transición tanto en Q. magnoliifolia (14\% en el sitio 3; Fig. 4a) como en $Q$. rugosa (9\%; Bonfil, 1998b). Esto muestra que en $Q$. liebmanii la consecuencia más importante de los incendios fue la disminución de tamaño de las categorías juveniles. La mortalidad se concentró en los mismos estadios: en el sitio 1 afectó a los Juveniles 1 y 2, mientras que en el sitio 2 se presentó sólo en los primeros (figura 2). Las diferencias en la mortalidad y en los valores de las transiciones de retrogresión apuntan a una mayor intensidad del incendio que se presentó en el sitio 1 respecto al ocurrido en el sitio 2.

$Q$. magnoliifolia mostró un comportamiento de respuesta al fuego similar al registrado en $Q$. liebmanii (figura 4). Nuevamente dominó la estasis en los árboles y las plántulas, que se mantuvieron en su misma categoría, y las transiciones correspondientes a la retrogresión fueron particularmente importantes en los estadios juveniles (Juveniles 2 en el sitio 3 y Juveniles 1 en el sitio 4). Las diferencias más importantes entre sitios probablemente se deban a variaciones en la intensidad de los incendios, ya que en el sitio 3 no se registró mortalidad e incluso se presentó una transición de crecimiento, mientras que en el sitio 4 sí hubo mortalidad de juveniles y no se registraron transiciones de crecimiento (figura 4b). Estas diferencias entre los sitios se confirman al comparar sus correspondientes distribuciones de frecuencias pre y postincendio: mientras en el sitio 3 ambas fueron muy similares, en el 4 se observó una disminución relativa de las categorías juveniles y un aumento de las plántulas (figura 3b), lo que se debió sobre todo al alto valor de la retrogresión Juveniles $1 \rightarrow$ plántulas. El impacto de esta retrogresión se amortiguó en términos numéricos, por la retrogresión Juveniles $2 \rightarrow$ Juveniles 1 , lo que explica que los cambios en la estructura poblacional no alcanzaran el nivel de significancia del 5\%.

Los análisis de la cobertura antes y después del incendio confirman la observación de que el impacto del fuego depende del estadio de tamaño en ambas especies (cuadro 5). Las plántulas sobreviven al fuego debido a que desarrollan una raíz pivotante que penetra profundamente en el suelo, en donde el aumento en la temperatura ocasionado por los incendios se atenúa rápidamente (Christensen, 1985). La muerte de la parte aérea estimula el desarrollo de las yemas 
latentes que se encuentran en la base del tallo, las cuales producen rebrotes que recuperan rápidamente la cobertura original, lo que explica la falta de efectos significativos del factor tiempo (i.e. incendio) en los análisis de varianza respectivos (cuadro 5). La alta capacidad de rebrotar, presente en las especies del género Quercus desde los primeros estadios de tamaño, ha sido reportada en varios estudios (Griffin 1980; Plumb 1980; Negreros y Snook 1984; Rodríguez 1996; Bonfil 1998a; Zavala et al., 2000). La rápida recuperación del follaje puede estar relacionada con el aprovechamiento de los nutrimentos ( $\mathrm{N}, \mathrm{K}, \mathrm{P}, \mathrm{Ca}, \mathrm{Mg}$ y $\mathrm{Na}$ ) liberados por el incendio (Ahlgren y Ahlgren, 1960; James y Smith, 1977; DeSouza et al., 1986). Reich et al. (1990) reportan en Quercus ellipsoidalis niveles foliares elevados de $\mathrm{N}, \mathrm{P}, \mathrm{K}$ y $\mathrm{Mg}$ en sitios incendiados. Las diferencias significativas debidas al sitio en Q. magnoliifolia (el sitio 4 presentó consistentemente plántulas con mayor cobertura que el sitio 3, figura 6a), pueden atribuirse a factores ambientales o a diferencias en la edad promedio, pues es evidente que en esta categoría coexisten individuos con diferencias de edad importantes, que por la altura de su parte aérea quedaron incluidos en la categoría de plántulas, tal como se ha reportado en otras especies de Quercus (Merz y Boyce, 1956).

La cobertura de los juveniles de ambas especies también resultó fuertemente afectada por los incendios, especialmente en el caso de los Juveniles 1 (factor tiempo en el cuadro 5), y aunque después del incendio se desarrollaron rebrotes vigorosos, en general no recuperaron la cobertura original. La recuperación fue desigual entre los sitios (figuras $5 \mathrm{~b}$ y $6 \mathrm{~b}$ ), aunque estas diferencias en general no fueron significativas. Los Juveniles 2 de Q. liebmanii recuperaron mejor su cobertura en el sitio 2 después del incendio que los del sitio 1 (figura 5c), lo que explica el efecto significativo de la interacción sitio x tiempo en el análisis de varianza respectivo. La rápida recuperación que se presentó en ambos estadios juveniles en el sitio 2 muestra que los rebrotes producidos después del incendio pueden alcanzar un crecimiento vigoroso en un plazo relativamente corto. En $Q$. magnoliifolia la falta de significancia en el análisis de la cobertura de los Juveniles 2 apoya la observación, surgida del diagrama del ciclo de vida, de que en esta especie los Juveniles 1 resultaron más afectados. Los primeros tuvieron una recuperación notable de su cobertura dos meses después del incendio (figura $6 \mathrm{c}$ ).

La cobertura de los adultos de ambas especies no se vio afectada por el fuego, lo que corrobora observaciones previas de que estos árboles soportan bien los incendios superficiales, ya que incluso si la copa se hubiera afectado parcialmente, su recuperación fue rápida. A partir de un diámetro de entre 13 y $16 \mathrm{~cm}$ (que es el DAP promedio de los árboles de $Q$. liebmanii y $Q$. magnoliifolia, respectivamente), los árboles de ambas especies pudieron enfrentar con éxito el impacto de los incendios. Estos valores son semejantes a los reportados por Plumb (1980) para Q. agrifolia, una especie nativa de
California, cuyos árboles deben poseer un DAP de al menos $15 \mathrm{~cm}$ para no ser dañados fuertemente por el fuego. Las diferencias significativas debidas al factor sitio en la cobertura de los adultos de ambas especies (cuadro 5) confirman que los árboles con diámetros más grandes, y por lo tanto más maduros, se localizan en los sitios 1 (Q. liebmanii) y 3 ( $Q$. magnoliifolia), mientras que los otros dos sitios contienen individuos de menor talla, probablemente más jóvenes. Sin embargo, para el caso de $Q$. liebmanii no puede descartarse que parte de la variación en la cobertura se deba a diferencias en la intensidad de los incendios, que se presentaron en distinta fecha en el sitio 1 y 2 (cuadro 2 ).

Los resultados del presente trabajo sugieren que, de continuar por un periodo prolongado el actual régimen de incendios de la zona, el reclutamiento de árboles adultos se vería seriamente limitado. Aunque la ausencia de datos de fecundidad de los adultos (y por lo tanto del ingreso de nuevos individuos a la población) impidió elaborar un modelo matricial, que permitiría analizar con más detalle la dinámica de las poblaciones de estas especies, es evidente (por la importancia de las transiciones de permanencia y retrogresión, y la muy pobre presencia de aquéllas correspondientes al crecimiento), que de continuar indefinidamente el presente régimen de incendios, las poblaciones de encinos se extinguirían, ya que una vez que los adultos hayan muerto no habría reclutamiento vía semillas, y la alta frecuencia de incendios impediría el crecimiento de los rebrotes hasta alcanzar la categoría de adultos. La mayor susceptibilidad de los juveniles al fuego probablemente se debe a que su corteza no tiene el grosor suficiente para impedir la muerte de los tejidos vivos, por lo que después de cada incendio el crecimiento de la parte aérea debe reiniciarse a partir de las yemas que se encuentran en la base del tallo. Cuando se producen nuevos rebrotes se movilizan las reservas contenidas en los tejidos subterráneos (raíz, y en su caso, lignotuber) de los encinos (Reich et al., 1980; Canadell et $a l ., 1999)$, y si este proceso se repite en intervalos cortos, dichas reservas pueden agotarse, provocando el debilitamiento y en última instancia la muerte del individuo.

De este análisis se desprende que si se pretende conservar a los encinos como elementos dominantes de las comunidades vegetales de la zona, es importante que se dé un control efectivo de los incendios. Aunque ambas especies tuvieron un comportamiento similar frente al fuego, lo que muestra que pueden tolerar los incendios superficiales y que tienen una alta resiliencia, el régimen de incendios actual puede poner en peligro la continuidad de sus poblaciones. Los resultados de este estudio coinciden con el punto de vista de Johnson et al. (2002), quienes reconocen que, a pesar de la estrecha relación entre encinos y fuego, el reclutamiento periódico de individuos al dosel requiere de intervalos sin fuego suficientemente largos, los cuales no se presentan en la zona de estudio actualmente. Queda por establecer cuál sería un intervalo adecuado para que se produzca el ingreso 
de individuos adultos a las poblaciones estudiadas.

En el contexto de comunidades indígenas pobres, como San José Lagunas, que dependen de la leña de encino como principal fuente de combustible para consumo doméstico, la falta de reclutamiento de individuos adultos a la población cobra una relevancia adicional. De continuar la presión para aprovechar los árboles adultos y la alta frecuencia de incendios, los bosques estudiados podrían transformarse en pinares. Se ha reportando que el disturbio antropogénico provoca la "pinarización" de bosques mixtos, ya que se favorece el reclutamiento, la supervivencia y el crecimiento de las especies de Pinus, pero no de las de Quercus (GonzálezEspinosa et al., 1995; López Barrera, 1998). Sin embargo, con una alta frecuencia de incendios sostenida, incluso la regeneración de los pinos podría verse amenazada, lo que contribuiría al deterioro que se presenta ya en extensiones importantes de la región de La Montaña (Landa et al., 1997).

\section{Agradecimientos}

Agradecemos especialmente a M. Ricker por su ayuda en el análisis de los datos. E. Jardel, M. Kappelle, J. Meave y un revisor anónimo hicieron sugerencias que mejoraron el manuscrito original. Los fondos para el trabajo de campo fueron proporcionados por el Programa de Aprovechamiento Integral de los Recursos Naturales, Universidad Nacional Autónoma de México.

\section{Literatura citada}

Abrams M.D. 1992. Fire and the development of oak forests. BioScience 42:346-353.

Abrams M.D. y Downs J.A. 1990. Successional replacement of oldgrowth white oak by mixed-mesophytic hardwoods in southwest Pennsylvania. Canadian Journal of Forest Research 20:1864-1870.

Ahlgren C.E. 1974. Effects of fires on temperate forests: North Central United States. En: Kozlowski T.T. y Ahlgren C.E. Eds. Fires and Ecosystems, pp. 195-223, Academic Press, Nueva York. Ahlgren I.F. y Ahlgren C.E. 1960. Ecological effects of forest fires. The Botanical Review 26:483-533.

Arias C.T. 1993. Manejo y consumo de leña en un municipio rural de subsistencia: Alcozauca, Guerrero. Tesis de Licenciatura, Facultad de Ciencias, Universidad Nacional Autónoma de México, México, D.F. 128 pp.

Bonfil C. 1998a. The effects of seed size, cotiledon reserves, and herbivory on seedlings survival and growth in Quercus rugosa and Quercus laurina (Fagaceae). American Journal of Botany 85:79-87.

Bonfil C. 1998b. Dinámica poblacional y regeneración de Quercus rugosa: implicaciones para la restauración de bosques de encinos. Tesis de Doctorado en Ecología. Instituto de Ecología, , Universidad Nacional Autónoma de México, México, D.F.

Bonfil C. y Valencia S. 1993. La riqueza de los encinos. Ciencias 29:13-15.

Bonfil C. y Soberón J. 1999. Quercus rugosa seedlings dynamics in relation to its re-introduction in a disturbed Mexican landscape. Applied Vegetation Science 2:189-200.

Bucley R. 1984. The role of fire. Response to Snyder. Oikos 43:405406.

Bratton S.P. y Miller S.G. 1994. History field system and the structure of maritime oak forest, Cumberland Island National Seashore, Georgia. Bulletin of the Torrey Botanical Club 121:1-12.

Canadell J., Djema A., López B., Lloret F., Sabaté S., Siscart D. y Gracia C.A. 1999. Structure and dynamics of the root system. En: Rodá F., Retana J., Gracia C.A. y Bellot J. Eds. Ecology of Mediterranean Evergreen Oak Forests, pp. 47-59, Ecological Studies 137, Springer, Berlín.

Christensen N.L. 1985. Shrubland fire regimes and their evolutionary consequences. En: Pickett S.T.A. y White P.S. Eds. The Ecology of Natural Disturbance and Patch Dynamics, pp. 85-100, Academic Press, Orlando, Florida.

Curtis J.T. y McIntosh R.P. 1951. An upland forest continuum in the prairie-forest border region of Wisconsin. Ecology 32:476496.

Crow T.R. 1988. Reproductive mode and mechanisms for self-replacement of northern red oak (Quercus rubra) - a review. Forest Science 34:19-40.

DeSouza J., Silka P.A. y Silva S.D. 1986. Comparative physiology of burned and unburned Rhus laurina after chaparral wild fire. Oecologia 71:63-68.

Fuller M. 1991. Forest fires: an introduction to wildlife behaviour, management, firefighting and prevention. Wiley Nature Editions, John Wiley \& Sons, Nueva York.

García E. 1987. Modificaciones al Sistema de Clasificación Climática de Köppen. Editado por la autora, México, D.F.

González-Espinosa M., Quintana-Ascencio P.F., Ramírez-Marcial N. y Gaytán-Guzmán P. 1991. Secondary succession in disturbed Pinus-Quercus forest in the highlands of Chiapas, México. Journal of Vegetation Science 2:351-360.

González-Espinosa M., Ramírez-Marcial N., Quintana-Ascencio P.F. y Martínez-Icó M. 1995. La utilización de los encinos y la conservación de la biodiversidad en Los Altos de Chiapas. Memorias del 3er Seminario Nacional sobre utilización de encinos. Facultad de Ciencias Forestales, Universidad Autónoma de Nuevo León, Linares, N.L.

González-Villareal L.M. 1986. Contribución al conocimiento del género Quercus (Fagaceae) en el Estado de Jalisco. Colección Flora de Jalisco. Instituto de Botánica, Universidad Autónoma de Guadalajara, Guadalajara.

Griffin J.R. 1980. Sprouting in fire-damage valley oaks, Chews Ridge, California. Proceedings of the Symposium on the Ecology, Management and Utilization of California Oaks, USDA General Technical Report PSW 44. Pacific Southwest Forest and Range Experimental Station, Claremont, California. pp. 216-219.

Grimm E.C. 1984. Fire and other factors controlling the Big Woods vegetation of Minnesota in the mid-nineteenth century. Ecological Monographs 54:291-311.

Haggerty P.K. 1991. Fire effects in blue oak woodland. En: Proceedings of the Symposium on Oak Woodland and Hardwood Rangeland Management, pp. 342-344, USDA General Technical Report PSW 126, Pacific Southwest Research Station, Berkeley.

INEGI. 1991. XI Censo General de Población y Vivienda 1990. Resultados Definitivos Guerrero. Tomos I, II, y III. Instituto Nacional de Estadística Geografía e Informática, Aguascalientes. 
James T.D.W. y Smith D.W. 1977. Short-term effects of surface fire on the biomass and nutrient standing crop of Populus tremuloides in southern Ontario. Canadian Journal of Forest Research 7:666-679.

Johnson P.S., Shifley S.R. y Roegers R. 2002. The Ecology and Silviculture of Oaks. CABI Publishing, Nueva York.

Keeley J.E. y Zedler P.H. 1998. Evolution of life histories in Pinus. En: Richardson D.M. Ed. Ecology and Biogeography of Pinus, pp. 219-249, Cambridge University Press, Cambridge.

Komarek E.V. 1974. Effects of fires on temperate forest and related ecosystems: Southeastern United States. En: Kozlowski T.T. y Ahlgren C.E. Eds. Fires and Ecosystems, pp. 251-277, Academic Press, Nueva York.

Kozlowski T.T. 1971. Growth and Development of Trees. Vol. II. Academic Press, Nueva York.

Kozlowski T.T., Kramer P.J. y Pallardy S.G. 1991. Fire. En: Kozlowski T.T. Ed. The Physiological Ecology of Woody Plants, pp. 401-424, Academic Press, San Diego.

Landa R., Meave J. y Carabias J. 1997. Environment deterioration in rural Mexico: an examination of the concept. Ecological Applications 7:316-329.

Lloret F., Retana J. y Espelta J.M. 1996. Efects dels focs i mecanismes de regeneració de les plantes. En: Terradas J. Ed. Ecologia del Foc, pp. 141-156, Ediciones Proa, Barcelona, España.

López Barrera F. 1998. Germinación y establecimiento temprano de Quercus rugosa y sus implicaciones en la rehabilitación de hábitats pinarizados en Los Altos de Chiapas, México. Tesis de Licenciatura, Facultad de Ciencias, Universidad Nacional Autónoma de México, México, D.F. 89 pp.

Lorimer C.G. 1985. The role of fire in the perpetuation of oak forests. En: Johnson J.E. Ed. Challenges in Oak Management and Utilization, pp. 8-25, Cooperative Extension Service, University of Wisconsin, Madison.

Masera O.R., Ordoñez M.J. y Dirzo R. 1997. Carbon emissions from Mexican forests: current situation and long-term scenarios. Climatic Change 35:265-295.

McCreary D.D., Tietje W.D., Schmidt R.H., Gross R., Weitkamp W.H., Willoughby B.L. y Bell F.L. 1991. Stump sprouting of blue oaks in California. En: Proceedings of the Symposium on Oak Woodland and Hardwood Rangeland Management, pp. 6469, USDA General Technical Report PSW 126, Pacific Southwest Research Station, Berkeley.

McGee G.G. 1995. Understory response to springtime prescribed fire in two New York transition oak forests. Forest Ecology and Management 76:149-168.

Meave del Castillo J. 1990. Estructura y Composición de la Selva Alta Perennifolia de los Alrededores de Bonampak. Instituto Nacional de Antropología e Historia, México, D.F.

Merz R.W. y Boyce S.G. 1956. Age of oak "seedlings". Journal of Forestry 54:774-775.

Negreros C.P. y Snook L. 1984. Análisis del efecto de la intensidad de corta sobre la regeneración natural de pinos en un bosque de pino-encino. Ciencia Forestal 47:48-61.

Nixon K.C. 1993a. The genus Quercus in Mexico. En: Ramarmoothy T.P., Bye R. y Fa J.E. Eds. Biological Diversity of Mexico, pp. 439-458, Oxford University Press, Oxford.

Nixon K.C. 1993b. Infrageneric classification of Quercus (Fagaceae) and typification of sectional names. Annales des Sciences Forestières 50, Suppl. 1: 25S-34S.
Obieta C. y Sarukhán K.J. 1981. Estructura y composición de la vegetación herbácea de un bosque uniespecífico de Pinus hartwegii. Boletín de la Sociedad Botánica de México 41:75123.

Perry P.J. 1991. The pines of Mexico and Central America. Timber Press, Portland.

Plumb T.R. 1980. Response of oaks to fire. Proceedings of the Symposium on the Ecology, Management and Utilization of California Oaks, USDA General Technical Report PSW 44, pp. 202215. Pacific Southwest Forest and Range Experimental Station, Claremont, California.

Quintana-Ascencio P., Espinoza M. y Ramírez-Marcial N. 1992. Acorn removal, seedling survivorship and seedling growth of Quercus crispipilis in successional forests of the highlands of Chiapas, Mexico. Bulletin of the Torrey Botanical Club 119:618.

Reich P.B, Teskey R.O., Johnson P.S. y Hinckley T.M. 1980. Periodic root and shoot growth in oaks. Forest Science 26:590-598.

Reich P.B., Abrams M.D., Ellsworth D.S., Kruger E.L. y Tabone T.J. 1990. Fire affects ecophysiology and community dynamics of central Wisconsin oak forest regeneration. Ecology 71:21792190.

Rebertus A.J., Williamson G.B. y Moser E.B. 1989. Longleaf pine pyrogenicity and turkey oak mortality in Florida xeric sandhills. Ecology 70:60-70.

Retana J. 1996. Caracteristíques de intensitat i extensio dels incendis. En: Terradas J. Ed. Ecologia del Foc, pp. 59-62, Ediciones Proa, Barcelona, España.

Rodríguez T.D.A. 1996. Incendios Forestales. Universidad Autónoma de Chapingo y Ed. Mundi Prensa, México, D.F.

Rzedowski J. 1978. Vegetación de México. Limusa, México, D.F.

Sánchez L.R. 1986. Estudio de la sucesión forestal en la sierra de Juárez, Oaxaca, México, después de un incendio forestal superficial. Biótica 11:219-231.

Sarukhán K.J. 1968. Análisis sinecológico de las selvas de Terminalia amazonia en la planicie costera del Golfo de México. Tesis de Maestría, Colegio de Posgraduados, Chapingo, Estado de México, 300 pp.

SEMARNAP. 1990. Incendios Forestales. SEMARNAP, México, D.F.

SEMARNAP. 1995. Incendios Forestales. SEMARNAP, México, D.F.

SEMARNAP. 1998. Incendios Forestales. SEMARNAP, México, D.F.

SEMARNAP. 1999. Incendios Forestales. SEMARNAP, México, D.F.

Sieg C.H. y Wright H.A. 1996. The role of prescribed burning in regenerating Quercus macrocarpa and associated woody plants in stringer woodlands in the Black Hills, South Dakota. International Journal of Wildland Fire 6:21-29.

Silvertown J., Franco M., Pisanty I., Mendoza A. 1993. Comparative plant demography: relative importance of life-cycle components to the finite rate of increase in woody and herbaceous perennials. Journal of Ecology 81: 465-476.

Sokal R.R. y Rohlf F.J. 1995. Biometry. W.H. Freeman and Company, Nueva York.

Sosa C.V., Cedeño O., Rodríguez E., Martínez R. y Raygoza A. 1999. Incendios Forestales. SEGOB y SEMARNAP, México, D.F.

Toledo C.A. 1994. Diagnóstico ecogeográfico y ordenamiento 
ambiental del municipio de Alcozauca, Guerrero a través de un SIG. Tesis de Maestría, Facultad de Ciencias, Universidad Nacional Autónoma de México, México, D.F. 207 pp.

Valiente-Banuet A., González M.F. y Piñero D. 1995. La vegetación selvática de la región de Gómez Farías, Tamaulipas, México. Acta Botanica Mexicana 33:1-36.

Valencia A.S. 1995. Contribución al género Quercus (Fagaceae) en el estado de Guerrero México. Facultad de Ciencias, Universidad Nacional Autónoma de México, México, D.F.

Viro P.J. 1974. Effects of forest fires on soil. En: Kozlowski T.T. y Ahlgren C.E. Eds. Fires and Ecosystems, pp. 7-45, Academic Press, Nueva York.

Williamson G.B. y Black E.M. 1981. High temperature of forest fires under pines as a selective advantage over oaks. Nature 293:643-644.

Fecha de recepción: 30 de abril de 2002

Versión corregida: 15 de abril de 2003

Aceptado: 15 de abril de 2003
Williams C.E. y Johnson W.C. 1990. Age structure and the maintenance of Pinus pungens in Pine-oak forests of southwestern Virginia. American Midland Naturalist 124:130-141.

Williams C.E. y Johnson W.C. 1992. Factors affecting recruitment of Pinus pungens in the southern Appalachian mountains. $\mathrm{Ca}$ nadian Journal Forest Research 22:878-887.

Zavala F. 1990. Los encinos mexicanos: un recurso desaprovechado. Ciencia y Desarrollo 95:43-51.

Zavala F. y García E. 1997. Plántulas y rebrotes en la regeneración de encinos en la sierra de Pachuca, Hidalgo. Agrociencia 31:323329.

Zavala M.A., Espelta J.M. y Retana J. 2000. Constraints and tradeoffs in Mediterranean plant communities: the case of Holm oakAleppo pine forests. Botanical Review 66:119-139. 